\title{
The direct health-care burden of valvular heart disease: evidence from US national survey data
}

\section{Matt Moore' \\ Jie Chen ${ }^{2}$ \\ Peter J Mallow ${ }^{3}$ \\ John A Rizzo ${ }^{4}$}

'Global Health Economic Strategy, Edwards Lifesciences Inc, Irvine, CA, ${ }^{2}$ Department of Health Services and Administration, University of Maryland, College Park, MD, ${ }^{3}$ Health Economics and Outcomes Research, CTI Clinical Trial \& Consulting Services Inc, Cincinnati, $\mathrm{OH},{ }^{4}$ Department of Preventive Medicine and Economics, Stony Brook University, Stony Brook, NY, USA
Correspondence: John A Rizzo

Department of Preventive Medicine and Economics, Stony Brook University, 100 Nicolls Road, Stony Brook, NY II790, USA

$\mathrm{Tel}+\mathrm{I} 6318286517$

Email rizzologic@gmail.com
This article was published in the following Dove Press journal:

ClinicoEconomics and Outcomes Research

18 October 2016

Number of times this article has been viewed

Purpose: This study quantified the overall effects of aortic valve disease (AVD) and mitral valve disease (MVD) by disease severity on direct health-care costs to insurers and patients.

Materials and methods: Based on 1996-2011 data from the Medical Expenditure Panel Survey (MEPS), a large, nationally representative US database, multivariate analyses were performed to assess the relationship between AVD and MVD and direct annual health-care costs to insurers and patients, at individual and US-aggregate levels. Adults aged 18 years and over with diagnosis codes for AVD or MVD based on International Classification of Diseases (ninth revision) diagnosis codes were identified. Subjects were further classified as symptomatic AVD, asymptomatic AVD, symptomatic MVD, and asymptomatic MVD. These classifications were determined with clinical assistance and based in part on data availability in the MEPS.

Results: The MEPS database included 148 patients with AVD: 53 patients with symptomatic AVD, 95 patients with asymptomatic AVD, and 1,051 with MVD, including 315 patients with symptomatic MVD and 736 patients with asymptomatic MVD. Symptomatic AVD had the largest incremental effect on annual per patient health-care expenditure: $\$ 12,789$ for symptomatic AVD, $\$ 10,816$ for asymptomatic AVD, $\$ 5,163$ for symptomatic MVD, and $\$ 1,755$ for asymptomatic MVD. When aggregated to the US population, heart-valve disease accounted for an incremental annual cost of $\$ 23.4$ billion. The largest aggregate annual costs were incurred by patients with symptomatic MVD ( $\$ 7.6$ billion), followed by symptomatic AVD ( $\$ 5.6$ billion), asymptomatic MVD ( $\$ 5.6$ billion), and asymptomatic AVD (\$4.6 billion).

Conclusion: The annualized incremental costs of heart-valve disease were substantial for all groups examined, and greatest for patients with symptomatic MVD. This reflects the relatively high prevalence associated with this group. With a growing and aging population, the prevalence of heart-valve disease is expected to rise, increasing the burden on public health.

Keywords: aortic valve disease, mitral valve disease, direct health-care costs, Medical Expenditure Panel Survey

\section{Introduction}

Valvular heart disease (VHD) is common in the US population, and is a disease that increases with age (particularly over 75 years). ${ }^{1}$ Mitral valve diseases (MVDs) and aortic valve diseases (AVDs) are the most common, and in both of these disease settings patients may present as symptomatic or asymptomatic. Mitral regurgitation (MR) and aortic stenosis (AS) account for the majority of diagnoses. ${ }^{1,2}$ Diseased heart valves are typically treated surgically with valve repair or valve replacement.

The natural history of VHD is most often characterized by a prolonged asymptomatic period that is frequently managed initially by primary care physicians who play 
a key role in diagnosis and timely referral for intervention; ${ }^{3}$ however, evidence suggests that many patients with severe VHD remain untreated, despite the existence of widely accepted practice guidelines developed by the American College of Cardiology (ACC) and the American Heart Association (AHA).$^{4-8}$ There are currently no drug therapies that can reverse or stop the progression of VHD. ${ }^{9,10}$

Approximately $45 \%$ of native VHD is aortic, and $2 \%-7 \%$ of people aged 65 years and older and about $13 \%$ of those aged over 75 years have a clinical diagnosis of symptomatic AS. ${ }^{10-13}$ Approximately 1.5 million people in the US suffer from AS, and about 500,000 within this group of patients suffer from severe AS. An estimated 250,000 patients with severe AS are symptomatic. ${ }^{14}$ Without a new valve, approximately half of these patients will die within 2-3 years. ${ }^{11,15}$

MR is the most frequent form of VHD in the US, and prevalence increases with age. ${ }^{12}$ The most common finding is leaflet prolapse, which results in varying degrees of $\mathrm{MV}$ regurgitation. ${ }^{4}$ Despite ACC/AHA recommendations for surgical intervention in adult patients with significant MR and preserved left ventricular function, many patients remain untreated, despite the risks of no intervention. Current estimates indicate that only one in 40 patients with moderate or severe MR undergoes surgical treatment. ${ }^{9,12,16,17}$

The clinical effectiveness and benefits of surgical interventions for VHD are well documented in the literature; however, the current literature provides limited evidence on the direct health-care costs of VHD and cost comparisons of AVD and MVD. ${ }^{9,12,18}$ Insurer expenditure and patient out-of-pocket (OOP) expenditure associated with VHD are not well understood either. Prior studies have focused on the elderly and the Medicare population, and provide little if any evidence on US aggregate health-care costs of VHD. Additionally, past studies have not controlled for a full array of confounding factors (ie, comorbidities and sociodemographic factors), and hence estimated effects of VHD may be biased. To bridge these gaps in the literature, this study generated individual and US aggregate estimates for the direct costs associated with VHD. As disease severity is likely to affect health-care costs, it is of interest to compare patients with symptomatic vs asymptomatic VHD. The present study makes the following contributions: 1) it controls for a wide range of potentially confounding comorbidities, reducing concerns about bias in the estimated effects of VHD; 2) it provides nationally representative estimates of the disease burden; 3) it includes separate estimates of insurer and OOP costs; and 4) it provides estimates for different types of VHD (eg, symptomatic and asymptomatic AVD and symptomatic and asymptomatic MVD).

\section{Materials and methods}

A retrospective analysis was conducted using the Medical Expenditure Panel Survey (MEPS), a large, nationally representative database developed by the Agency for Healthcare Research and Quality. The purpose was to quantify individual and US national estimates of health-care insurer expenditure and patient OOP expenditure associated with VHD for four cohorts categorized according to valve-disease type (aortic or mitral) and symptom status (symptomatic or asymptomatic). This study is a retrospective database analysis where de-identified data were accessed in compliance with the Health Insurance Portability and Accountability Act. As a retrospective analysis of a de-identified database, the research was exempt from IRB review and informed patient consent under 45 CFR 46.101(b)(4).

\section{Data source}

This study used data from the 1996-2011 MEPS, a subset of the National Health Interview Survey, which includes information on health-care utilization and expenditure, health status, health insurance coverage, and sociodemographic and socioeconomic characteristics for the civilian, noninstitutionalized population in the US. ${ }^{19}$ MEPS, a nationally representative database, is one of the most widely used databases for quantifying health-care expenditure and laborproductivity costs. An electronic search of the Agency for Healthcare Research and Quality (www.ahrq.gov) generated over 1,000 journal publications to date. The survey on which MEPS data is based utilizes a complex survey design that includes clustering and oversampling of certain subgroups, such as minorities. ${ }^{20}$ MEPS collects comprehensive data on individuals and their health-care expenditure and use over a span of roughly 2 years, with response rates varying from approximately $60 \%$ to $80 \%$. Household-survey data are collected by means of computer-assisted personal interviews, with data supplemented by information collected directly from the medical providers used by survey participants. Insurance data are collected both from households and through a separate survey of employers' business establishments, which collect information on health insurance provided by US employers. Health-care expenditure data in the MEPS are self-reported; however, medical providers help to validate the self-reported data and resolve inconsistencies when they occur.

\section{Study sample}

Adults aged 18 years and over with diagnosis codes for AVD or MVD based on the International Classification of Diseases (ICD)-9 diagnosis codes were identified (see Table S1 for a list of the relevant codes). Subjects were further classified 
as symptomatic AVD, asymptomatic AVD, symptomatic MVD, and asymptomatic MVD. These classifications were determined with clinical assistance and based in part on information on comorbidities in MEPS (see Table S2 for a list of these comorbidities).

The sample included in this study consisted of 148 patients with AVD: 53 patients with symptomatic AVD and 95 patients with asymptomatic AVD. An additional 1,051 patients were identified with MVD, including 315 patients with symptomatic MVD and 736 patients with asymptomatic MVD. A comparison group of 259,491 subjects without heart-valve disease was included to enable us to estimate the impacts of AVD and MVD on direct health-care costs (Figure 1).

\section{Dependent variable: medical expenditure}

The expenditure data contained in the MEPS includes spending on physicians, hospital and outpatient services, medications, diagnostic testing, and other medical services. This study used total annual expenditure on these various health-care services, and categorized total expenditure according to the amounts paid by health-care insurers and patients' OOP payments.

\section{Explanatory variables: clinical and sociodemographic characteristics}

This study included a number of explanatory variables affecting expenditure, including major chronic diseases, sociodemographics, geographic region, and year. Chronic diseases were measured as binary variables (1, disease present; 0 , disease absent), and included the four heart-valve disease cohorts along with 30 other major chronic illnesses. These diseases were chosen based on their prevalence and clinical considerations. A wide array of comorbidities were included, because previous research has shown that failure to control adequately for comorbidities may lead to substantial upward bias in the estimated expenditure impact of the disease of interest. ${ }^{21}$
Sociodemographic factors included age strata, education, race, income, marital status, and health insurance type. Race variables included African-American, Hispanic, Asian, and other, with Caucasian serving as the reference group. Insurance status was measured as a series of binary indicators: uninsured, Medicaid, Medicare, other public insurance, private non-health maintenance organization insurance, and private health maintenance organization insurance (reference group). We also included variables indicating whether the subject was interviewed in English and whether the subject had a usual source of care. "Usual source of care" is the particular medical professional, doctor's office, clinic, health center, or other place where a person would typically go if sick or in need of advice about his or her health. Geographic variables included census region (Midwest, South, West, and Northeast, with Northeast serving as the reference region) and whether a patient resided in a metropolitan statistical area. Year was measured as a series of binary variables, with 1996 serving as the reference year. Further descriptions of these variables are included in the tables of results.

\section{Statistical methods}

Two-part models were estimated in which the likelihood of incurring any expenditure and the conditional expenditure were estimated separately by logistic and ordinary least squares models, respectively. ${ }^{22}$ Specifically, the analysis estimated the likelihood that a patient had positive medical expenditure, and separate conditional expenditure models were constructed for OOP expenses and insurer expenditure. The two-part model is frequently used in health economics research when many observations are clustered and the remaining observations are skewed to the right. ${ }^{23}$ To normalize the distribution of the error terms, expenditure was log-transformed.

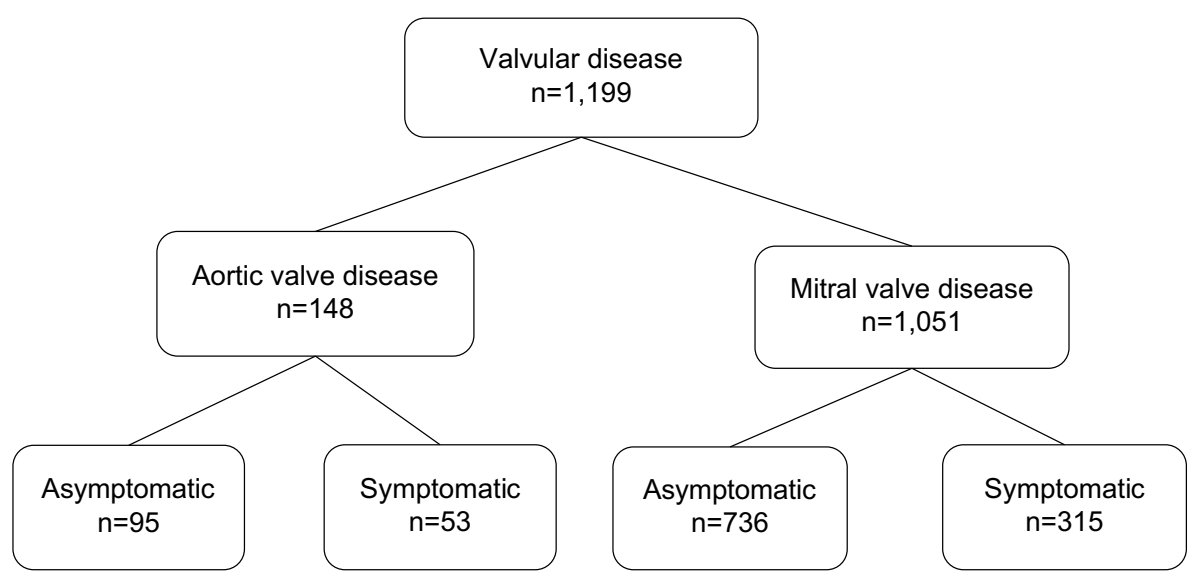

Figure I Patients identified in MEPS 1996-20II.

Abbreviations: MEPS, Medical Expenditure Panel Survey; ICD, International Classification of Diseases. 
The logistic regression models were written as:

PrExpenditure $=\alpha_{0}+\alpha_{1} \mathrm{AVD}+\alpha_{2} \mathrm{AVD}_{\text {symp }}$ $+\alpha_{3} \mathrm{MVD}+\alpha_{4} \mathrm{MVD}_{\text {symp }}+\beta$ Comorbidities $+\Theta \mathbf{X}+\varepsilon$

where PrExpenditure is a binary variable equal to 1 if medical expenses are positive and 0 otherwise; AVD and $\mathrm{AVD}_{\text {symp }}$ are binary variables indicating whether the subject has asymptomatic or symptomatic AVD, respectively; MVD and MVD $_{\text {symp }}$ are binary variables indicating whether the subject has asymptomatic or symptomatic MVD, respectively; "comorbidities" denotes a vector of binary indicator variables for the presence or absence of other diseases; $\mathrm{X}$ is a vector of sociodemographic, economic, region, and year variables; $\alpha_{0}-\alpha_{4}, \beta$, and $\Theta$ are coefficients to be estimated; and $\varepsilon$ is the error term. Equation 1 was estimated separately to ascertain the probabilities of health-care insurer and OOP expenditure. The effects of each valvular disease cohort on expenditure were measured relative to adult subjects without valvular disease, who served as the reference group.

In the second stage, conditional expenditure models were estimated by the following equation:

lnExpenditure $=\alpha_{0}+\alpha_{1} \mathrm{AVD}+\alpha_{2} \mathrm{AVD}_{\text {symp }}+$

$\alpha_{3}$ MVD $+\alpha_{4}$ MVD $_{\text {symp }}+\beta$ Comorbidities $+\Theta \mathbf{X}+\varepsilon$

where lnExpenditure is the natural logarithm of conditional health-care expenditure and other terms are as for Equation 1. The models were again estimated separately for health-care insurer and OOP expenditure. Expenditures were adjusted to 2011 US dollars using the "medical care" component of the Consumer Price Index. ${ }^{24}$ All models were estimated using Stata version 11 (StataCorp LP, College Station, TX,USA).

\section{Sensitivity analysis}

The effects of disease on the burden of illness are sensitive to model specifications and disease prevalence. Additionally, patients with more severe disease may be systematically more likely to indicate the presence of disease. Therefore, the self-reported disease measure may bias incremental expenditure estimates upward. On the other hand, random measurement error in the disease-indicator variable may bias the incremental expenditure estimates downward. ${ }^{25}$ Therefore, to address these sources of uncertainty and to gauge the robustness of the results to alternative assumptions, sensitivity analyses were performed by varying the prevalence rate of valvular disease from baseline $\pm 25 \%$. Also, the estimated incremental expenditure effects of VHD were varied by $25 \%$ above and below for the corresponding base-case expenditure.

\section{Results}

\section{Descriptive statistics}

Descriptive statistics for the study sample are provided in Table 1. The total sample comprised 148 subjects with AVD, of whom 53 were symptomatic and 1,051 with MVD, of whom 736 were asymptomatic. The reference group without VHD comprised 259,491 subjects. AVD patients were older and more likely to be male compared to both MVD and reference-group cohorts. Among both AVD and MVD groups, subjects with symptomatic aortic disease were older. Not surprisingly, the reference group was younger than either

Table I Descriptive statistics for aortic and mitral valve disease and no-valvular-disease cohorts

\begin{tabular}{|c|c|c|c|c|c|}
\hline \multirow[t]{3}{*}{ Variable names } & \multirow{2}{*}{$\begin{array}{l}\text { No valvular } \\
\text { disease }\end{array}$} & \multicolumn{2}{|c|}{ Aortic valve disease } & \multicolumn{2}{|c|}{ Mitral valve disease } \\
\hline & & Symptomatic & Asymptomatic & Symptomatic & Asymptomatic \\
\hline & $n=259,491$ & $n=53$ & $n=95$ & $n=315$ & $n=736$ \\
\hline \multicolumn{6}{|l|}{ Direct health-care expenditure } \\
\hline Any insurer expenditure & $84 \%$ & $100 \%$ & $100 \%$ & $99 \%$ & $96 \%$ \\
\hline Any out-of-pocket expenditure & $88 \%$ & $100 \%$ & $97 \%$ & $99 \%$ & $99 \%$ \\
\hline Insurer expenditure, if any & $\$ 5,302$ & $\$ 27,128$ & $\$ 13,357$ & $\$ 11,875$ & $\$ 5,694$ \\
\hline Out-of-pocket expenditure, if any & $\$ 1,033$ & $\$ 3,018$ & $\$ 2,708$ & $\$ 2,180$ & $\$ 1,493$ \\
\hline \multicolumn{6}{|l|}{ Demographics } \\
\hline Age, years, mean & 47.1 & 67.4 & 60.7 & 57.3 & 52.2 \\
\hline $18-24$ & $11 \%$ & 0 & $7 \%$ & $3 \%$ & $4 \%$ \\
\hline $25-34$ & $17 \%$ & $2 \%$ & $4 \%$ & $4 \%$ & $9 \%$ \\
\hline $35-44$ & $19 \%$ & 0 & $4 \%$ & I I\% & $21 \%$ \\
\hline $45-54$ & $19 \%$ & $17 \%$ & $17 \%$ & $26 \%$ & $25 \%$ \\
\hline $55-64$ & $15 \%$ & $19 \%$ & $19 \%$ & $19 \%$ & $18 \%$ \\
\hline $65-74$ & $10 \%$ & $19 \%$ & $23 \%$ & $23 \%$ & $15 \%$ \\
\hline $75+$ & $9 \%$ & $43 \%$ & $25 \%$ & $14 \%$ & $9 \%$ \\
\hline
\end{tabular}


Table I (Continued)

\begin{tabular}{|c|c|c|c|c|c|}
\hline \multirow[t]{3}{*}{ Variable names } & \multirow{2}{*}{$\begin{array}{l}\text { No valvular } \\
\text { disease }\end{array}$} & \multicolumn{2}{|c|}{ Aortic valve disease } & \multicolumn{2}{|c|}{ Mitral valve disease } \\
\hline & & Symptomatic & Asymptomatic & Symptomatic & Asymptomatic \\
\hline & $n=259,491$ & $n=53$ & $n=95$ & $n=315$ & $n=736$ \\
\hline Sex, female & $57 \%$ & $55 \%$ & $39 \%$ & $83 \%$ & $81 \%$ \\
\hline \multicolumn{6}{|l|}{ Race/ethnicity } \\
\hline Caucasian & $60 \%$ & $91 \%$ & $84 \%$ & $89 \%$ & $86 \%$ \\
\hline Hispanic & $20 \%$ & $2 \%$ & $7 \%$ & $5 \%$ & $5 \%$ \\
\hline African-American & $15 \%$ & $8 \%$ & $7 \%$ & $5 \%$ & $7 \%$ \\
\hline Asian & $4 \%$ & 0 & $1 \%$ & $0 \%$ & $1 \%$ \\
\hline Other & $1 \%$ & 0 & 0 & $1 \%$ & $1 \%$ \\
\hline Marital status, married & $51 \%$ & $55 \%$ & $63 \%$ & $55 \%$ & $58 \%$ \\
\hline \multicolumn{6}{|l|}{ Education } \\
\hline No high school & $28 \%$ & $36 \%$ & $20 \%$ & $18 \%$ & $12 \%$ \\
\hline High school degree & $44 \%$ & $36 \%$ & $41 \%$ & $41 \%$ & $44 \%$ \\
\hline Some college & $14 \%$ & $13 \%$ & $24 \%$ & $15 \%$ & $21 \%$ \\
\hline College or advanced & $14 \%$ & $15 \%$ & $15 \%$ & $26 \%$ & $22 \%$ \\
\hline \multicolumn{6}{|l|}{ Income/employment status } \\
\hline Family income $<100 \% \mathrm{FPL}$ & $16 \%$ & $23 \%$ & $12 \%$ & $12 \%$ & $9 \%$ \\
\hline Family income $100 \%-199 \%$ FPL & $21 \%$ & $25 \%$ & $20 \%$ & $13 \%$ & $12 \%$ \\
\hline Family income $\geq 200 \% \mathrm{FPL}$ & $64 \%$ & $53 \%$ & $68 \%$ & $75 \%$ & $79 \%$ \\
\hline \multicolumn{6}{|l|}{ Health insurance* } \\
\hline Uninsured & $14 \%$ & $2 \%$ & $3 \%$ & $3 \%$ & $5 \%$ \\
\hline Medicaid & $13 \%$ & $11 \%$ & $11 \%$ & $10 \%$ & $4 \%$ \\
\hline Medicare & $11 \%$ & $25 \%$ & $16 \%$ & $16 \%$ & $9 \%$ \\
\hline Other public health insurance & $1 \%$ & 0 & 0 & 0 & $1 \%$ \\
\hline Private non-HMO insurance & $39 \%$ & $60 \%$ & $53 \%$ & $55 \%$ & $55 \%$ \\
\hline Private $\mathrm{HMO}$ insurance & $26 \%$ & $8 \%$ & $21 \%$ & $20 \%$ & $28 \%$ \\
\hline \multicolumn{6}{|l|}{ Health status } \\
\hline Physical health: fair or poor & $18 \%$ & $49 \%$ & $15 \%$ & $34 \%$ & $16 \%$ \\
\hline Physical health: good & $29 \%$ & $15 \%$ & $31 \%$ & $27 \%$ & $30 \%$ \\
\hline Physical health: very good or excellent & $53 \%$ & $36 \%$ & $55 \%$ & $39 \%$ & $55 \%$ \\
\hline Mental health: fair or poor & $8 \%$ & $25 \%$ & $2 \%$ & $14 \%$ & $7 \%$ \\
\hline Mental health: good & $26 \%$ & $32 \%$ & $28 \%$ & $28 \%$ & $24 \%$ \\
\hline Mental health: very good or excellent & $66 \%$ & $43 \%$ & $69 \%$ & $58 \%$ & $70 \%$ \\
\hline Access to usual source of health care & $81 \%$ & $96 \%$ & $94 \%$ & $94 \%$ & $92 \%$ \\
\hline Interviewed in English & $89 \%$ & $98 \%$ & $97 \%$ & $99 \%$ & $99 \%$ \\
\hline Location, urban & $81 \%$ & $83 \%$ & $78 \%$ & $76 \%$ & $81 \%$ \\
\hline \multicolumn{6}{|l|}{ US census regions } \\
\hline Northeast & $16 \%$ & $26 \%$ & $17 \%$ & $18 \%$ & $21 \%$ \\
\hline Midwest & $22 \%$ & $23 \%$ & $24 \%$ & $22 \%$ & $24 \%$ \\
\hline South & $38 \%$ & $38 \%$ & $37 \%$ & $43 \%$ & $39 \%$ \\
\hline West & $25 \%$ & $13 \%$ & $22 \%$ & $17 \%$ & $17 \%$ \\
\hline \multicolumn{6}{|l|}{ Year } \\
\hline 2011 & $7 \%$ & $8 \%$ & $7 \%$ & $7 \%$ & $5 \%$ \\
\hline 2010 & $7 \%$ & $11 \%$ & $4 \%$ & $7 \%$ & $6 \%$ \\
\hline 2009 & $7 \%$ & $8 \%$ & $7 \%$ & $8 \%$ & $7 \%$ \\
\hline 2008 & $7 \%$ & 0 & $5 \%$ & $8 \%$ & $6 \%$ \\
\hline 2007 & $6 \%$ & $6 \%$ & $5 \%$ & $5 \%$ & $6 \%$ \\
\hline 2006 & $7 \%$ & $6 \%$ & $3 \%$ & $7 \%$ & $6 \%$ \\
\hline 2005 & $7 \%$ & $13 \%$ & $7 \%$ & $9 \%$ & $6 \%$ \\
\hline 2004 & $7 \%$ & $11 \%$ & $15 \%$ & $8 \%$ & $7 \%$ \\
\hline 2003 & $7 \%$ & $9 \%$ & $8 \%$ & $9 \%$ & $7 \%$ \\
\hline 2002 & $8 \%$ & $6 \%$ & $14 \%$ & $9 \%$ & $8 \%$ \\
\hline 2001 & $7 \%$ & $6 \%$ & $8 \%$ & $4 \%$ & $8 \%$ \\
\hline 2000 & $5 \%$ & $4 \%$ & $3 \%$ & $3 \%$ & $4 \%$ \\
\hline 1999 & $5 \%$ & $4 \%$ & $3 \%$ & $4 \%$ & $6 \%$ \\
\hline 1998 & $5 \%$ & $6 \%$ & $4 \%$ & $5 \%$ & $7 \%$ \\
\hline 1997 & $3 \%$ & $2 \%$ & $2 \%$ & $3 \%$ & $4 \%$ \\
\hline 1996 & $5 \%$ & $2 \%$ & $2 \%$ & $3 \%$ & $6 \%$ \\
\hline
\end{tabular}


Table I (Continued)

\begin{tabular}{|c|c|c|c|c|c|}
\hline \multirow[t]{3}{*}{ Variable names } & \multirow{2}{*}{$\begin{array}{l}\text { No valvular } \\
\text { disease }\end{array}$} & \multicolumn{2}{|c|}{ Aortic valve disease } & \multicolumn{2}{|c|}{ Mitral valve disease } \\
\hline & & Symptomatic & Asymptomatic & Symptomatic & Asymptomatic \\
\hline & $n=259,491$ & $n=53$ & $n=95$ & $n=315$ & $n=736$ \\
\hline \multicolumn{6}{|c|}{ Comorbid medical condition (\%) } \\
\hline \multicolumn{6}{|c|}{ Malignant neoplasms } \\
\hline Skin cancer & $2 \%$ & $8 \%$ & $8 \%$ & $8 \%$ & $3 \%$ \\
\hline Colon cancer & 0 & 0 & 0 & $1 \%$ & 0 \\
\hline Lung cancer & 0 & 0 & 0 & $1 \%$ & 0 \\
\hline Bladder cancer & 0 & $2 \%$ & 0 & $1 \%$ & 0 \\
\hline Rectal cancer & 0 & 0 & 0 & 0 & 0 \\
\hline \multicolumn{6}{|c|}{ Diseases of the digestive system } \\
\hline GERD & $6 \%$ & $21 \%$ & $6 \%$ & $19 \%$ & $11 \%$ \\
\hline Gastritis & $1 \%$ & 0 & $2 \%$ & $2 \%$ & $1 \%$ \\
\hline Gastric ulcer & $1 \%$ & $2 \%$ & 0 & $3 \%$ & $1 \%$ \\
\hline Diverticulitis & $1 \%$ & 0 & $2 \%$ & $3 \%$ & $2 \%$ \\
\hline \multicolumn{6}{|c|}{ Diseases of the genitourinary system } \\
\hline Kidney stones & $1 \%$ & $4 \%$ & 0 & $2 \%$ & $1 \%$ \\
\hline Cystitis & $2 \%$ & $4 \%$ & 0 & $5 \%$ & $4 \%$ \\
\hline \multicolumn{6}{|l|}{ Mental disorders } \\
\hline Depressive disorders & $10 \%$ & $25 \%$ & $7 \%$ & $24 \%$ & $13 \%$ \\
\hline Neurotic disorders & $6 \%$ & $21 \%$ & $8 \%$ & $27 \%$ & $13 \%$ \\
\hline \multicolumn{6}{|c|}{ Diseases of the circulatory system } \\
\hline Coronary artery disease & $2 \%$ & $11 \%$ & $8 \%$ & $11 \%$ & $3 \%$ \\
\hline Congestive heart failure & $1 \%$ & $11 \%$ & $1 \%$ & $4 \%$ & $1 \%$ \\
\hline Acute myocardial infarction & $1 \%$ & $11 \%$ & $5 \%$ & $5 \%$ & $2 \%$ \\
\hline Stroke & 0 & 0 & $1 \%$ & $1 \%$ & 0 \\
\hline Cardiac dysrhythmia & $2 \%$ & $23 \%$ & $6 \%$ & $16 \%$ & $7 \%$ \\
\hline \multicolumn{6}{|c|}{$\begin{array}{l}\text { Diseases of the musculoskeletal } \\
\text { system and connective tissue }\end{array}$} \\
\hline Osteoarthritis & $1 \%$ & $2 \%$ & $1 \%$ & $3 \%$ & $1 \%$ \\
\hline Low-back pain & $9 \%$ & $23 \%$ & $6 \%$ & $15 \%$ & $11 \%$ \\
\hline Osteoporosis & $3 \%$ & $11 \%$ & $3 \%$ & $16 \%$ & $7 \%$ \\
\hline \multicolumn{6}{|c|}{ Diseases of the nervous system } \\
\hline Parkinson's disease & 0 & 0 & 0 & $1 \%$ & $1 \%$ \\
\hline Multiple sclerosis & 0 & 0 & 0 & $1 \%$ & 0 \\
\hline Migraine headache & $3 \%$ & 0 & 0 & $13 \%$ & $5 \%$ \\
\hline \multicolumn{6}{|c|}{ Diseases of the respiratory system } \\
\hline Bronchitis & $1 \%$ & $8 \%$ & 0 & $5 \%$ & $1 \%$ \\
\hline Emphysema & $1 \%$ & $6 \%$ & $4 \%$ & $2 \%$ & $0 \%$ \\
\hline Asthma & $6 \%$ & $17 \%$ & $3 \%$ & $13 \%$ & $9 \%$ \\
\hline \multicolumn{6}{|l|}{ Metabolic diseases } \\
\hline Diabetes & $10 \%$ & $11 \%$ & $4 \%$ & $16 \%$ & $6 \%$ \\
\hline High cholesterol & $16 \%$ & $47 \%$ & $37 \%$ & $36 \%$ & $21 \%$ \\
\hline Hypothyroidism & $3 \%$ & $8 \%$ & $3 \%$ & $10 \%$ & $9 \%$ \\
\hline
\end{tabular}

Note: *Possible to have more than one insurance type. Descriptive statistics for all explanatory variables are in proportions.

Abbreviations: FPL, Federal Poverty Level; HMO, health maintenance organization; GERD, gastroesophageal reflux disease.

VHD cohort. Symptomatic aortic disease patients were more likely to have depressive and neurotic disorders than asymptomatic aortic disease patients, as well as more diseases of the circulatory system. Both AVD and MVD groups were more likely to be Caucasian and to have health insurance compared to subjects in the reference group.

Symptomatic AVD subjects had insurer expenditure $(\$ 27,128)$ that was over twice that of asymptomatic AVD patients $(\$ 13,357)$. Insurer expenditure for symptomatic
MVD patients was also over twice that of asymptomatic MVD patients ( $\$ 11,875$ vs $\$ 5,694)$. OOP expenditure was also greater for symptomatic aortic disease patients than for asymptomatic patients, though the differences were less pronounced.

\section{Multivariable results}

Table 2 presents the logistic regression estimates for the likelihood of incurring insurer and OOP expenditure. Patients with 
Table 2 Logistic regression of the probabilities of positive health-care insurer cost and positive OOP costs (per annum)

\begin{tabular}{|c|c|c|c|c|}
\hline \multirow[t]{2}{*}{ Explanatory variables } & \multicolumn{2}{|l|}{ Total OOP } & \multicolumn{2}{|c|}{ Total insurer cost } \\
\hline & Odds ratio & $P$-value & Odds ratio & $P$-value \\
\hline \multicolumn{5}{|l|}{ Type of valvular disease } \\
\hline No valve disease & Reference group & & Reference group & \\
\hline Aortic valve disease, symptomatic* & - & & - & \\
\hline Aortic valve disease, asymptomatic* & 3.14 & 0.1 & - & \\
\hline Mitral valve disease, symptomatic & 3.12 & 0.13 & 3.68 & 0.03 \\
\hline Mitral valve disease, asymptomatic & 6.81 & 0 & 2.19 & 0 \\
\hline \multicolumn{5}{|l|}{ Medical conditions } \\
\hline \multicolumn{5}{|l|}{ Malignant neoplasms } \\
\hline Skin cancer & 2.43 & 0 & 4.14 & 0 \\
\hline Colon cancer & 2.73 & 0.01 & 4.3 & 0 \\
\hline Lung cancer & 1.28 & 0.42 & 12.74 & 0 \\
\hline Bladder cancer* & - & & 33.62 & 0 \\
\hline Rectal cancer & 0.84 & 0.8 & 0.61 & 0.49 \\
\hline \multicolumn{5}{|l|}{ Diseases of the digestive system } \\
\hline GERD & 3.71 & 0 & 3.91 & 0 \\
\hline Gastritis & 2.76 & 0 & 1.83 & 0 \\
\hline Gastric ulcer & 1.59 & 0 & 1.97 & 0 \\
\hline Diverticulitis & 2.79 & 0 & 2.64 & 0 \\
\hline \multicolumn{5}{|l|}{ Diseases of the genitourinary system } \\
\hline Kidney stones & 2.7 & 0 & 2.36 & 0 \\
\hline Cystitis & 4.21 & 0 & 2.34 & 0 \\
\hline \multicolumn{5}{|l|}{ Mental disorders } \\
\hline Depressive disorders & 1.53 & 0 & 1.44 & 0 \\
\hline Neurotic disorders & 2.12 & 0 & 1.84 & 0 \\
\hline \multicolumn{5}{|l|}{ Diseases of the circulatory system } \\
\hline Coronary artery disease & 1.14 & 0.34 & 1.95 & 0 \\
\hline Congestive heart failure & 6.93 & 0 & 3.53 & 0 \\
\hline Acute myocardial infarction & 2.13 & 0 & 2.59 & 0 \\
\hline Stroke & 4.31 & 0.01 & 8.73 & 0 \\
\hline Cardiac dysrhythmia & 4.13 & 0 & 3.08 & 0 \\
\hline \multicolumn{5}{|c|}{ Diseases of the musculoskeletal system and connective tissue } \\
\hline Osteoarthritis & 1.5 & 0.03 & 1.27 & 0.07 \\
\hline Low-back pain & 1.29 & 0 & 1.33 & 0 \\
\hline Osteoporosis & 2.99 & 0 & 2.99 & 0 \\
\hline \multicolumn{5}{|l|}{ Diseases of the nervous system } \\
\hline Parkinson's disease & 3.65 & 0 & 4.3 & 0 \\
\hline Multiple sclerosis & 3.66 & 0 & 2.3 & 0.04 \\
\hline Migraine headache & 1.56 & 0 & 1.46 & 0 \\
\hline \multicolumn{5}{|l|}{ Diseases of the respiratory system } \\
\hline Bronchitis & 1.6 & 0 & 1.39 & 0.01 \\
\hline Emphysema & 1.06 & 0.7 & 1.69 & 0 \\
\hline Asthma & 2.3 & 0 & 1.88 & 0 \\
\hline \multicolumn{5}{|l|}{ Metabolic diseases } \\
\hline Diabetes & 3.72 & 0 & 2.44 & 0 \\
\hline High cholesterol & 3.03 & 0 & 3.03 & 0 \\
\hline Hypothyroidism & 10.68 & 0 & 2.48 & 0 \\
\hline \multicolumn{5}{|l|}{ Health status } \\
\hline Physical health: fair or poor & Reference group & & Reference group & \\
\hline Physical health: good & 0.77 & 0 & 0.77 & 0 \\
\hline Physical health: very good or excellent & 0.66 & 0 & 0.66 & 0 \\
\hline Mental health: fair or poor & Reference group & & Reference group & \\
\hline Mental health: good & 1.01 & 0.89 & 0.92 & 0.05 \\
\hline Mental health: very good or excellent & 1.08 & 0.05 & I & 0.94 \\
\hline \multicolumn{5}{|l|}{ Race/ethnicity } \\
\hline Caucasian & Reference group & & Reference group & \\
\hline Hispanic & 0.72 & 0 & 0.83 & 0 \\
\hline African-American & 0.67 & 0 & 0.93 & 0.01 \\
\hline
\end{tabular}


Table 2 (Continued)

\begin{tabular}{|c|c|c|c|c|}
\hline \multirow[t]{2}{*}{ Explanatory variables } & \multicolumn{2}{|l|}{ Total OOP } & \multicolumn{2}{|c|}{ Total insurer cost } \\
\hline & Odds ratio & $P$-value & Odds ratio & $P$-value \\
\hline Asian & 0.67 & 0 & 0.63 & 0 \\
\hline Other & 0.51 & 0 & 1.06 & 0.57 \\
\hline \multicolumn{5}{|l|}{ Age, years } \\
\hline $18-24$ & \multicolumn{2}{|c|}{ Reference group } & \multicolumn{2}{|c|}{ Reference group } \\
\hline $25-34$ & 0.98 & 0.56 & 0.88 & 0 \\
\hline $35-44$ & 0.98 & 0.46 & 0.87 & 0 \\
\hline $45-54$ & 1.29 & 0 & 0.96 & 0.15 \\
\hline $55-64$ & 1.62 & 0 & 1.08 & 0.03 \\
\hline $65-74$ & 1.92 & 0 & 1.56 & 0 \\
\hline $75+$ & 2.2 & 0 & 2.55 & 0 \\
\hline Sex, female & 2.06 & 0 & 1.76 & 0 \\
\hline Marital status, married & 1.1 & 0 & 1.27 & 0 \\
\hline \multicolumn{5}{|l|}{ Education } \\
\hline No high school & \multicolumn{2}{|c|}{ Reference group } & \multicolumn{2}{|c|}{ Reference group } \\
\hline High school & 1.2 & 0 & 1.14 & 0 \\
\hline Some college & 1.94 & 0 & 1.68 & 0 \\
\hline College or advanced & 1.77 & 0 & 1.66 & 0 \\
\hline \multicolumn{5}{|l|}{ Income/employment status } \\
\hline Family income $<100 \% \mathrm{FPL}$ & \multicolumn{2}{|c|}{ Reference group } & \multicolumn{2}{|c|}{ Reference group } \\
\hline Family income $100 \%-199 \% \mathrm{FPL}$ & 0.7 & 0 & 0.84 & 0 \\
\hline Family income $\geq 200 \% \mathrm{FPL}$ & 0.78 & 0 & 0.78 & 0 \\
\hline Unemployed & 1.01 & 0.58 & 1.17 & 0 \\
\hline \multicolumn{5}{|l|}{ Health insurance } \\
\hline Private non-HMO insurance & \multicolumn{2}{|c|}{ Reference group } & \multicolumn{2}{|c|}{ Reference group } \\
\hline Uninsured & 0.45 & 0 & 0.08 & 0 \\
\hline Medicaid & 0.37 & 0 & $\mathrm{I} .43$ & 0 \\
\hline Medicare & 1.14 & 0.01 & 0.68 & 0 \\
\hline Other public health insurance & 1.58 & 0 & 0.77 & 0.01 \\
\hline Private $\mathrm{HMO}$ insurance & 1.02 & 0.37 & 0.88 & 0 \\
\hline Access to usual source of health care & 2.52 & 0 & 2.75 & 0 \\
\hline Interviewed in English & 1.05 & 0.18 & 1.13 & 0 \\
\hline Location, urban & 0.94 & 0.02 & 1.11 & 0 \\
\hline \multicolumn{5}{|l|}{ US census regions } \\
\hline Northeast & \multicolumn{2}{|c|}{ Reference group } & \multicolumn{2}{|c|}{ Reference group } \\
\hline Midwest & 1.01 & 0.71 & 0.9 & 0 \\
\hline South & 1.12 & 0 & 0.84 & 0 \\
\hline West & 0.89 & 0 & 0.87 & 0 \\
\hline Year & & & & \\
\hline 1996 & Reference gro & & Reference gr & \\
\hline 2011 & 0.89 & 0.02 & 1.41 & 0 \\
\hline 2010 & 0.81 & 0 & 1.51 & 0 \\
\hline 2009 & 0.85 & 0 & 1.49 & 0 \\
\hline 2008 & 0.94 & 0.22 & 1.49 & 0 \\
\hline 2007 & 1.01 & 0.79 & $\mathrm{I} .44$ & 0 \\
\hline 2006 & 1.02 & 0.72 & 1.3 & 0 \\
\hline 2005 & 1.08 & 0.15 & 1.36 & 0 \\
\hline 2004 & 0.99 & 0.84 & 1.32 & 0 \\
\hline 2003 & 1.13 & 0.01 & 1.37 & 0 \\
\hline 2002 & 1.13 & 0.01 & 1.39 & 0 \\
\hline 2001 & 1.21 & 0 & 1.34 & 0 \\
\hline 2000 & 1.21 & 0 & 1.30 & 0 \\
\hline 1999 & 1.18 & 0 & 1.35 & 0 \\
\hline 1998 & 1.06 & 0.3 & 1.13 & 0.02 \\
\hline 1997 & 1.07 & 0.35 & 1.08 & 0.20 \\
\hline Intercept term & 2.75 & 0 & 2.08 & 0 \\
\hline
\end{tabular}

Notes: *Symptomatic aortic valve disease and bladder cancer had perfect correlation with OOP expenditure, and were dropped from the analysis. Similarly, symptomatic and asymptomatic aortic valve disease had perfect correlation with insurer expenditure, and so were excluded from the insurer expenditure regression. Abbreviations: FPL, Federal Poverty Level; HMO, health maintenance organization; GERD, gastroesophageal reflux disease; OOP, out of pocket. 
symptomatic MVD had a significantly higher likelihood of insurer expenditure than the reference group (odds ratio 3.68, $P<0.05$ ), as did asymptomatic MVD patients (odds ratio 2.19, $P<0.01)$. Because all AVD subjects had insurer expenditure, these cohorts were perfectly collinear with expenditure, and hence were dropped from the insurer regressions. Subjects with asymptomatic MVD were significantly more likely to incur OOP expenditure relative to the reference group (odds ratio 6.81, $P<0.01)$. All symptomatic AVD subjects incurred at least some OOP expenditure, and thus were excluded from the OOP estimation.

The odds of incurring insurer and OOP expenditure were generally higher for older subjects and those in worse health, as indicated by their comorbidity profiles and self-reported health status. Subjects with more education and having a usual source of care were more likely to incur insurer and OOP expenditure. African-Americans, Hispanics, and Asians were less likely to incur health-care insurer and OOP expenditure than Caucasian patients.

Using the logistic regression results from Table 2, predicted values of the likelihood of incurring insurer and OOP expenditure were obtained for each cohort. These results, provided in Table 3 , indicated that AVD substantially increases the likelihood of incurring expenditure. Subjects with symptomatic or asymptomatic AVD were 16 percentage points more likely to incur insurer expenditure compared to the reference group. Subjects with symptomatic MVD were 9 percentage points more likely to incur such expenditure, while asymptomatic MVD subjects were 6 percentage points more likely. The differences were smaller for OOP expenditure, though all valvular disease cohorts were more likely to incur this expenditure.

Conditional regression models were also estimated for all cohorts to estimate the effects of AVD on health-care expenditure for patients incurring at least some positive expenditure. These models included the same explanatory variables as in Table 2. The results, provided in Table 4, revealed that all four VHD cohorts had significantly higher conditional expenditure than the reference group. Comorbidities, worse self-reported health, and older age were all associated with higher conditional expenditure. Better-educated individuals and females had higher expenditure. Minorities tended to have lower conditional expenditure.

The regression model results (Tables 2 and 4) were used to estimate expected incremental per patient expenditure associated with VHD for each cohort. These results are provided in Table 5. Having symptomatic AVD increased annual health-care insurer expenditure by $\$ 12,022$, while asymptomatic disease raised this expenditure by $\$ 10,110$. Symptomatic MVD raised insurer expenditure by $\$ 4,718$, while asymptomatic disease increased this expenditure by $\$ 1,360$. OOP expenditure was higher for each VHD cohort in comparison to the reference group, although the differences were more modest.

\section{Aggregate expenditure and sensitivity analysis}

Table 6 shows US aggregate expenditure for each VHD cohort. US expenditure for each cohort was obtained by multiplying the incremental expenditure in Table 5 with the estimated aggregate number of patients in each group. The aggregate number of patients in each disease cohort was determined by multiplying their respective prevalence rates estimated from the MEPS with a US-population estimate for subjects aged 18 years and above, which was 191.1 million. ${ }^{26}$ The assumed baseline prevalence rate for VHD was $2.5 \%$, based on Nkomo et al. ${ }^{12}$ We multiplied this overall prevalence by the relative shares of the symptomatic AVD (8.5\%), asymptomatic AVD (8.1\%), symptomatic MVD (28.5\%), and asymptomatic MVD groups $(54.9 \%)$, respectively, in the MEPS data to obtain prevalence rates for each of these subgroups. Base-case estimates of expenditure and prevalence rates were varied plus or minus $25 \%$ in the sensitivity analysis.

Aggregate US expenditure for each cohort is provided in Table 6. VHD increased overall aggregate annual healthcare expenditure by $\$ 5.6$ billion, $\$ 4.6$ billion, $\$ 7.6$ billion, and $\$ 5.6$ billion in the symptomatic AVD, asymptomatic AVD, symptomatic MVD, and asymptomatic MVD groups, respectively. The overall total incremental expenditure for VHD was thus estimated as $\$ 23.4$ billion.

Table 3 Predicted probability of incurring expenditures (per annum)

\begin{tabular}{|c|c|c|c|c|c|}
\hline \multirow[t]{2}{*}{ Expenditure category } & \multirow[t]{2}{*}{ No valvular disease } & \multicolumn{2}{|c|}{ Aortic valve disease } & \multicolumn{2}{|c|}{ Mitral valve disease } \\
\hline & & Symptomatic & Asymptomatic & Symptomatic & Asymptomatic \\
\hline Insurer expenditure & $84 \%$ & $100 \%$ & $100 \%$ & $93 \%$ & $90 \%$ \\
\hline Difference compared to no valvular disease* & & 16 & 16 & 9 & 6 \\
\hline Out-of-pocket expenditure & $88 \%$ & $100 \%$ & $95 \%$ & $95 \%$ & $98 \%$ \\
\hline Difference compared to no valvular disease* & & 12 & 7 & 7 & 10 \\
\hline
\end{tabular}

Note: *These differences are percentage points compared to no valvular disease. 
Table 4 Ordinary least squares estimates of total health-care insurer cost and total out-of-pocket costs

\begin{tabular}{|c|c|c|c|c|}
\hline \multirow[t]{2}{*}{ Explanatory variables } & \multicolumn{2}{|c|}{ Total out of pocket, if any } & \multicolumn{2}{|c|}{ Total insurer cost, if any } \\
\hline & Coefficient & $P$-value & Coefficient & $P$-value \\
\hline \multicolumn{5}{|l|}{ Type of valvular disease } \\
\hline No valve disease & \multicolumn{2}{|c|}{ Reference group } & \multicolumn{2}{|c|}{ Reference group } \\
\hline Aortic valve disease, symptomatic & 0.47 & 0.01 & 0.99 & 0 \\
\hline Aortic valve disease, asymptomatic & 0.44 & 0 & 0.88 & 0 \\
\hline Mitral valve disease, symptomatic & 0.30 & 0 & 0.51 & 0 \\
\hline Mitral valve disease, asymptomatic & 0.27 & 0 & 0.17 & 0 \\
\hline \multicolumn{5}{|l|}{ Medical conditions } \\
\hline \multicolumn{5}{|l|}{ Malignant neoplasms } \\
\hline Skin cancer & 0.19 & 0 & 0.41 & 0 \\
\hline Colon cancer & 0.17 & 0.01 & 0.95 & 0 \\
\hline Lung cancer & 0.26 & 0 & 1.23 & 0 \\
\hline Bladder cancer & 0.29 & 0 & 0.63 & 0 \\
\hline Rectal cancer & 0.27 & 0.25 & 0.97 & 0 \\
\hline \multicolumn{5}{|l|}{ Diseases of the digestive system } \\
\hline GERD & 0.31 & 0 & 0.54 & 0 \\
\hline Gastritis & 0.29 & 0 & 0.45 & 0 \\
\hline Gastric ulcer & 0.27 & 0 & 0.49 & 0 \\
\hline Diverticulitis & 0.17 & 0 & 0.53 & 0 \\
\hline \multicolumn{5}{|l|}{ Diseases of the genitourinary system } \\
\hline Kidney stones & 0.27 & 0 & 0.79 & 0 \\
\hline Cystitis & 0.21 & 0 & 0.24 & 0 \\
\hline \multicolumn{5}{|l|}{ Mental disorders } \\
\hline Depressive disorders & 0.37 & 0 & 0.39 & 0 \\
\hline Neurotic disorders & 0.33 & 0 & 0.32 & 0 \\
\hline \multicolumn{5}{|l|}{ Diseases of the circulatory system } \\
\hline Coronary artery disease & 0.05 & 0.03 & 0.27 & 0 \\
\hline Congestive heart failure & 0.29 & 0 & 0.67 & 0 \\
\hline Acute myocardial infarction & 0.24 & 0 & 0.7 & 0 \\
\hline Stroke & 0.27 & 0 & 0.65 & 0 \\
\hline Cardiac dysrhythmia & 0.29 & 0 & 0.52 & 0 \\
\hline \multicolumn{5}{|c|}{$\begin{array}{l}\text { Diseases of the musculoskeletal system and } \\
\text { connective tissue }\end{array}$} \\
\hline Osteoarthritis & 0.2 & 0 & 0.31 & 0 \\
\hline Low-back pain & 0.2 & 0 & 0.23 & 0 \\
\hline Osteoporosis & 0.29 & 0 & 0.3 & 0 \\
\hline \multicolumn{5}{|l|}{ Diseases of the nervous system } \\
\hline Parkinson's disease & 0.47 & 0 & 0.52 & 0 \\
\hline Multiple sclerosis & 0.74 & 0 & 1.04 & 0 \\
\hline Migraine headache & 0.29 & 0 & 0.26 & 0 \\
\hline \multicolumn{5}{|l|}{ Diseases of the respiratory system } \\
\hline Bronchitis & -0.08 & 0.01 & 0.07 & 0.09 \\
\hline Emphysema & 0.04 & 0.22 & 0.25 & 0 \\
\hline Asthma & 0.3 & 0 & 0.31 & 0 \\
\hline \multicolumn{5}{|l|}{ Metabolic diseases } \\
\hline Diabetes & 0.47 & 0 & 0.45 & 0 \\
\hline High cholesterol & 0.33 & 0 & 0.33 & 0 \\
\hline Hypothyroidism & 0.26 & 0 & 0.12 & 0 \\
\hline \multicolumn{5}{|l|}{ Health status } \\
\hline Physical health: fair or poor & Reference gro & & Reference gr & \\
\hline Physical health: good & -0.22 & 0 & -0.42 & 0 \\
\hline Physical health: very good or excellent & -0.39 & 0 & -0.7 & 0 \\
\hline Mental health: fair or poor & Reference grc & & Reference gr & \\
\hline Mental health: good & -0.07 & 0 & -0.09 & 0 \\
\hline Mental health: very good or excellent & -0.04 & 0.01 & -0.04 & 0.05 \\
\hline \multicolumn{5}{|l|}{ Race/ethnicity } \\
\hline Caucasian & Reference gro & & Reference gr & \\
\hline Hispanic & -0.27 & 0 & -0.14 & 0 \\
\hline
\end{tabular}


Table 4 (Continued)

\begin{tabular}{|c|c|c|c|c|}
\hline \multirow[t]{2}{*}{ Explanatory variables } & \multicolumn{2}{|c|}{ Total out of pocket, if any } & \multicolumn{2}{|c|}{ Total insurer cost, if any } \\
\hline & Coefficient & $P$-value & Coefficient & $P$-value \\
\hline African-American & -0.42 & 0 & -0.08 & 0 \\
\hline Asian & -0.4 & 0 & -0.32 & 0 \\
\hline Other & -0.27 & 0 & 0.15 & 0 \\
\hline \multicolumn{5}{|l|}{ Age, years } \\
\hline $18-24$ & \multicolumn{2}{|c|}{ Reference group } & \multicolumn{2}{|c|}{ Reference group } \\
\hline $25-34$ & 0.05 & 0 & 0.12 & 0 \\
\hline $35-44$ & 0.15 & 0 & 0.13 & 0 \\
\hline $45-54$ & 0.42 & 0 & 0.29 & 0 \\
\hline $55-64$ & 0.66 & 0 & 0.48 & 0 \\
\hline $65-74$ & 0.69 & 0 & 0.55 & 0 \\
\hline $75+$ & 0.78 & 0 & 0.7 & 0 \\
\hline Sex, female & 0.3 & 0 & 0.22 & 0 \\
\hline Marital status, married & -0.07 & 0 & 0.05 & 0 \\
\hline \multicolumn{5}{|l|}{ Education } \\
\hline No high school & \multicolumn{2}{|c|}{ Reference group } & \multicolumn{2}{|c|}{ Reference group } \\
\hline High school & 0.14 & 0 & 0.09 & 0 \\
\hline Some college & 0.36 & 0 & 0.19 & 0 \\
\hline College or advanced & 0.33 & 0 & 0.22 & 0 \\
\hline \multicolumn{5}{|l|}{ Income/employment status } \\
\hline Family income $<100 \% \mathrm{FPL}$ & \multicolumn{2}{|c|}{ Reference group } & \multicolumn{2}{|c|}{ Reference group } \\
\hline Family income $100 \%-199 \% \mathrm{FPL}$ & -0.23 & 0 & -0.05 & 0 \\
\hline Family income $\geq 200 \% \mathrm{FPL}$ & -0.13 & 0 & -0.08 & 0 \\
\hline Unemployed & 0.13 & 0 & 0.22 & 0 \\
\hline Access to usual source of health care & 0.18 & 0 & 0.31 & 0 \\
\hline \multicolumn{5}{|l|}{ Health insurance } \\
\hline Private non-HMO insurance & \multicolumn{2}{|c|}{ Reference group } & \multicolumn{2}{|c|}{ Reference group } \\
\hline Uninsured & 0.39 & 0 & -0.78 & 0 \\
\hline Medicaid & -1.06 & 0 & 0.38 & 0 \\
\hline Medicare & 0.21 & 0 & -0.12 & 0 \\
\hline Other public health insurance & 0.34 & 0 & -0.14 & 0 \\
\hline Private $\mathrm{HMO}$ insurance & 0.21 & 0 & 0.03 & 0.01 \\
\hline Interviewed in English & 0.16 & 0 & 0.23 & 0 \\
\hline Location, urban & 0 & 0.88 & 0.09 & 0 \\
\hline \multicolumn{5}{|l|}{ US census regions } \\
\hline Northeast & \multicolumn{2}{|c|}{ Reference group } & \multicolumn{2}{|c|}{ Reference group } \\
\hline Midwest & 0.08 & 0 & 0 & 0.98 \\
\hline South & 0.17 & 0 & -0.11 & 0 \\
\hline West & 0.06 & 0 & -0.06 & 0 \\
\hline Year & & & & \\
\hline 1996 & Reference gro & & Reference gr & \\
\hline 2011 & -0.45 & 0 & 0.05 & 0.06 \\
\hline 2010 & -0.43 & 0 & 0.06 & 0.03 \\
\hline 2009 & -0.37 & 0 & 0.11 & 0 \\
\hline 2008 & -0.28 & 0 & 0.09 & 0 \\
\hline 2007 & -0.2 & 0 & 0.16 & 0 \\
\hline 2006 & -0.06 & 0.01 & 0.13 & 0 \\
\hline 2005 & -0.02 & 0.43 & 0.15 & 0 \\
\hline 2004 & 0 & 0.84 & 0.18 & 0 \\
\hline 2003 & 0.06 & 0.01 & 0.15 & 0 \\
\hline 2002 & 0.01 & 0.81 & 0.15 & 0 \\
\hline 2001 & 0 & 0.96 & 0.16 & 0 \\
\hline 2000 & -0.1 & 0 & 0.06 & 0.05 \\
\hline 1999 & -0.12 & 0 & 0.06 & 0.06 \\
\hline 1998 & -0.1 & 0 & 0.02 & $0.4 I$ \\
\hline 1997 & -0.05 & 0.08 & 0.03 & 0.39 \\
\hline Intercept term & 5.13 & 0 & 6.2 & 0 \\
\hline
\end{tabular}

Abbreviations: GERD, gastroesophageal reflux disease; FPL, Federal Poverty Level; HMO, health maintenance organization. 
Table 5 Expected per-patient health-care expenditure (per annum)*

\begin{tabular}{|c|c|c|c|c|c|}
\hline \multirow[t]{2}{*}{ Expenditure category } & \multirow[t]{2}{*}{ No valvular disease } & \multicolumn{2}{|c|}{ Aortic valve disease } & \multicolumn{2}{|c|}{ Mitral valve disease } \\
\hline & & Symptomatic & Asymptomatic & Symptomatic & Asymptomatic \\
\hline Insurer expenditure & $\$ 7,133$ & $\$ 19,155$ & $\$ 17,242$ & $\$ 11,851$ & $\$ 8,493$ \\
\hline $\begin{array}{l}\text { Difference compared to no } \\
\text { valvular disease }\end{array}$ & & $\$ 12,022$ & $\$ 10,110$ & $\$ 4,718$ & $\$ 1,360$ \\
\hline Out-of-pocket expenditure & $\$ 1,263$ & $\$ 2,030$ & $\$ 1,969$ & $\$ 1,708$ & $\$ 1,658$ \\
\hline $\begin{array}{l}\text { Difference compared to no } \\
\text { valvular disease }\end{array}$ & & $\$ 767$ & $\$ 706$ & $\$ 445$ & $\$ 395$ \\
\hline
\end{tabular}

Note: *Calculated as estimated probability of incurring expenditure multiplied by conditional expenditure.

Table 6 Aggregate expenditure (US\$ billion per annum)

\begin{tabular}{|c|c|c|c|}
\hline \multirow{2}{*}{$\begin{array}{l}\text { Valvular heart disease } \\
\text { category }\end{array}$} & \multicolumn{3}{|c|}{ Prevalence rate } \\
\hline & Low & Base case & High \\
\hline \multicolumn{4}{|c|}{ Aortic valve disease, symptomatic vs no valvular disease } \\
\hline Prevalence rate & $0.16 \%$ & $0.213 \%$ & $0.266 \%$ \\
\hline High (baseline $+25 \%$ ) & $\$ 5.6$ & $\$ 7$ & $\$ 8.8$ \\
\hline Base case & $\$ 4.2$ & $\$ 5.6$ & $\$ 7$ \\
\hline Low (baseline $-25 \%$ ) & $\$ 3.2$ & $\$ 4.2$ & $\$ 5.3$ \\
\hline \multicolumn{4}{|c|}{ Aortic valve disease, asymptomatic vs no valvular disease } \\
\hline Prevalence rate & $0.145 \%$ & $0.193 \%$ & $0.241 \%$ \\
\hline High (baseline $+25 \%$ ) & $\$ 4.4$ & $\$ 5.8$ & $\$ 7.3$ \\
\hline Base case & $\$ 3.5$ & $\$ 4.6$ & $\$ 5.8$ \\
\hline Low (baseline -25\%) & $\$ 2.6$ & $\$ 3.5$ & $\$ 4.4$ \\
\hline \multicolumn{4}{|c|}{ Mitral valve disease, symptomatic vs no valvular disease } \\
\hline Prevalence rate & $0.533 \%$ & $0.711 \%$ & $0.889 \%$ \\
\hline High (baseline $+25 \%$ ) & $\$ 7.1$ & $\$ 9.5$ & $\$ 11.9$ \\
\hline Base case & $\$ 5.7$ & $\$ 7.6$ & $\$ 9.5$ \\
\hline Low (baseline $-25 \%$ ) & $\$ 4.3$ & $\$ 5.7$ & $\$ 7.1$ \\
\hline \multicolumn{4}{|c|}{ Mitral valve disease, asymptomatic vs no valvular disease } \\
\hline Prevalence rate & $1.037 \%$ & $1.383 \%$ & $1.729 \%$ \\
\hline High (baseline $+25 \%$ ) & $\$ 5.3$ & $\$ 7$ & $\$ 8.8$ \\
\hline Base case & $\$ 4.2$ & $\$ 5.6$ & $\$ 7$ \\
\hline Low (baseline $-25 \%$ ) & $\$ 3.2$ & $\$ 4.2$ & $\$ 5.3$ \\
\hline
\end{tabular}

Note: These aggregated expenditures used prevalence rates of MEPS and weighted population $(191,115,050)$ in MEPS.

Abbreviation: MEPS, Medical Expenditure Panel Survey.

The sensitivity analysis demonstrated that annual healthcare expenditure ranged from $\$ 3.2$ billion to $\$ 8.8$ billion in the symptomatic AVD group and $\$ 2.6$ billion to $\$ 7.3$ billion in the asymptomatic AVD cohort. The ranges for symptomatic MVD and asymptomatic MVD cohorts were \$4.3-\$11.9 billion and \$3.2-\$8.8 billion, respectively.

\section{Discussion}

This study found that each of four VHD cohorts had significantly higher insurer and OOP expenditure than health-care expenditure for similar individuals without VHD. In multivariate analyses that adjusted for a large number of potential confounders, the annual total incremental per patient health-care expenditure ranged from a low of $\$ 1,755$ in the asymptomatic MVD patient cohort to a high of $\$ 12,789$ in the symptomatic AVD group. Higher expenditure in the symptomatic AVD group was likely related to the surgical intervention of AV replacement (AVR) in this group. While per patient AVD costs were higher than for the MVD cohort, the aggregate costs of MVD ( $\$ 13.2$ billion) exceeded those for AVD ( $\$ 10.2$ billion). This reflects the considerably higher prevalence of MVD patients.

There is much debate in the literature regarding timing of both referral and surgical intervention in asymptomatic patients and whether early surgical intervention improves outcomes. ${ }^{4,27}$ While the new guidelines established by the ACC and the AHA advocate earlier intervention for asymptomatic patients, ${ }^{9}$ not all patients with VHD are diagnosed and treated. ${ }^{3,12}$ Nkomo et al found there was a difference in the prevalence of VHD in the US population and the percentage of people diagnosed with VHD in the community ( $2.5 \%$ vs $1.8 \%$ ), likely due to undiagnosed VHD. ${ }^{12}$

The literature suggests that undertreatment may result from an overestimation of operative risk, underestimation of symptoms, or misclassification of hemodynamic severity. ${ }^{28}$ Long-term clinical outcome results have established that medical management is often ineffective. In patients with asymptomatic MR and AS, "watchful waiting" for symptoms may be too late. ${ }^{4,29,30}$ Research has also demonstrated that AVR is a cost-effective alternative to medical management in most elderly patients. ${ }^{31}$ When curative, effective valve surgery relieves the patient of the symptom burden and thus the health-care burden of the unaddressed valve disease, making it a cost-effective solution.

Health-care expenditure in the symptomatic AVD and MVD cohorts may increase in the future as the share of the US population aged 65 years and over continues to grow. Advances in technology may increase the attractiveness of more aggressive treatment than medical management alone, as this offers the potential to improve patient outcomes. Unlike medical management, for example, surgical intervention offers the potential for curative treatment and longer survival. ${ }^{32}$ 
In our study, VHD increased overall aggregate annual health-care expenditure by $\$ 5.6$ billion, $\$ 4.6$ billion, $\$ 7.6$ billion, and $\$ 5.6$ billion in the symptomatic AVD, asymptomatic AVD, symptomatic MVD, and asymptomatic MVD groups, respectively. The overall total incremental expenditure for VHD was thus estimated at $\$ 23.4$ billion. For each of these cohorts, the percentage of patients over age 65 years was $62 \%, 48 \%, 37 \%$, and $24 \%$, respectively, with the largest elderly population in the symptomatic AVD and asymptomatic AVD groups. Collectively, the sum of health-care expenditure among the over-65-year population totals $\$ 12.2$ billion, or more than half the total expenditure for VHD. That a relatively larger percentage of elderly patients with symptomatic and asymptomatic AVD is not surprising: present patients with MVD tend to be younger. Given the rising numbers of elderly patients undergoing AVR, the disproportionate share of the economic burden of VHD for this population is likely to rise.

To our knowledge, this is the first study to provide nationally representative estimates of the direct health-care expenditure for VHD by type of valve disease (AVD or MVD) and symptomatic status. The sample design mitigates concerns about generalizability of the results by employing a sample that was nationally representative. By including a sensitivity analysis using a range of heart valve-disease prevalence estimates, this study addressed the tendency for prevalence rates to be underreported in surveys, a major methodological challenge recognized in survey research. ${ }^{33}$ In addition, this study included 30 relevant comorbid diseases, which helped control for potential confounding effects. Finally, the two-part model methodology is a widely accepted method of estimating expenditure in the econometrics literature, because it adjusts for patients who have no expenditure. ${ }^{23,34}$ If this adjustment is not made, estimates of incremental health-care expenditure associated with a disease are less reliable. ${ }^{23,34}$ We also gained on-site access to the restricted MEPS files, rather than using the publicly available standard MEPS data. This allowed the research team to obtain more detailed ICD-9 code information, which improved stratification of disease severity.

\section{Limitations}

This study has important limitations that must be noted. First, relevant factors that are related to health-care expenditure may have been omitted. However, given that the study included 30 comorbidities and a number of sociodemographic factors, the effect of any omissions may have been attenuated. Second, this study was based on self-reported data collected as part of a national household survey. Therefore, there was the potential for respondent-recall bias. The MEPS survey design attenuates recall bias through validation with physicians and insurers, however. Finally, we used a number of indicator conditions to proxy for whether the patient would be regarded as being symptomatic, and any proxy measure is subjected to some error. However, the pattern of higher expenditure for symptomatic vs asymptomatic patients is consistent with prior expectations.

\section{Conclusion}

Valvular disease is a significant public health concern in the US. The costs of VHD are substantial for each of the four cohorts we examined, and are greatest for MVD patients. This reflects the relatively high prevalence of MVD patients. Per capita health-care expenditure is greatest for the symptomatic AVD cohort, however. With a growing and aging population, the prevalence of VHD is expected to rise, further increasing the public health burden of the disease. Effective valve surgery relieves the patient of the symptom burden and thus the health-care burden of the unaddressed valve disease, making it an appealing treatment alternative. Future studies should continue to analyze shifting patterns in the clinical and economic burden of VHD, as well as the value of innovative treatment options to provide a more informed basis for health-policy decision-making.

\section{Acknowledgments}

The abstract of this paper was presented at ISPOR, May 19, 2015 in Philadelphia, PA as a poster presentation with interim findings. The abstract was published in Value in Health. Two additional abstracts of this paper were presented at AHA QCOR, March 1, 2016 in Phoenix, AZ as a poster presentation with interim findings. The abstract was published in Circulation: Cardiovascular Quality and Outcomes.

\section{Disclosure}

MM is an employee of Edwards Lifesciences Inc, the study sponsor. At the time of the study, PJM was an employee of CTI Clinical Trial and Consulting Services Inc. JAR is an academic affiliate of CTI, which is a consultant to Edwards Lifesciences Inc. JC reports no conflicts of interest in this work.

\section{References}

1. Roger VL, Go AS, Lloyd-Jones DM, et al. Heart disease and stroke statistics - 2011 update: a report from the American Heart Association. Circulation. 2011;123(4):e18-e209.

2. Ray S. Changing epidemiology and natural history of valvular heart disease. Clin Med. 2010;10(2):168-171. 
3. Helms AS, Bach DS. Heart valve disease. Prim Care. 2013;40(1): 91-108.

4. Adams DH, Rosenhek R, Falk V. Degenerative mitral valve regurgitation: best practice revolution. Eur Heart J. 2010;31(16):1958-1966.

5. Bach DS, Siao D, Girard SE, Duvernoy C, McCallister BD Jr, Gualano SK. Evaluation of patients with severe symptomatic aortic stenosis who do not undergo aortic valve replacement: the potential role of subjectively overestimated operative risk. Circ Cardiovasc Qual Outcomes. 2009;2(6):533-539.

6. Jan F, Andreev M, Mori N, Janosik B, Sagar K. Unoperated patients with severe symptomatic aortic stenosis. Circulation. 2009;120 Suppl 18:S753.

7. Freed BH, Sugeng L, Furlong K, et al. Reasons for nonadherence to guidelines for aortic valve replacement in patients with severe aortic stenosis and potential solutions. Am J Cardiol. 2010;105(9):1339-1342.

8. Gaibazzi N, Reverberi C, Ghillani M, Brunazzi B, Faggiano P. Prevalence of undiagnosed asymptomatic aortic valve stenosis in the general population older than 65 years: a screening strategy using cardiac auscultation followed by Doppler-echocardiography. Int J Cardiol. 2013;168(5):4905-4906.

9. Nishimura RA, Otto CM, Bonow RO, et al. 2014 AHA/ACC guideline for the management of patients with valvular heart disease - executive summary: a report of the American College of Cardiology/American Heart Association Task Force on Practice Guidelines. Circulation. 2014;129(23):2440-2492.

10. Vahanian A, Alfieri O, Andreotti F, et al. Guidelines on the management of valvular heart disease (version 2012): the Joint Task Force on the Management of Valvular Heart Disease of the European Society of Cardiology (ESC) and the European Association for Cardio-Thoracic Surgery (EACTS). Eur J Cardiothorac Surg. 2012;42(4):S1-S44.

11. Iung B, Baron G, Butchart EG, et al. A prospective survey of patients with valvular heart disease in Europe: the Euro Heart Survey on Valvular Heart Disease. Eur Heart J. 2003;24(13):1231-1243.

12. Nkomo VT, Gardin JM, Skelton TN, Gottdiener JS, Scott CG, EnriquezSarano M. Burden of valvular heart diseases: a population-based study. Lancet. 2006;368(9540):1005-1011.

13. Iung $B$, Vahanian $A$. Epidemiology of valvular heart disease in the adult. Nat Rev Cardiol. 2011;8(3):162-172.

14. John Muir Health. US aortic stenosis disease prevalence and treatment statistics. Available from: https://www.johnmuirhealth.com/services/ cardiovascular-services/intervention/transcatheter-aortic-valve-replacement/facts-and-figures.html. Accessed October 22, 2015.

15. Bonow RO, Carabello BA, Chatterjee K, et al. 2008 Focused update incorporated into the ACC/AHA 2006 guidelines for the management of patients with valvular heart disease: a report of the American College of Cardiology/American Heart Association Task Force on Practice Guidelines (writing committee to revise the 1998 guidelines for the management of patients with valvular heart disease). Circulation. 2008;118(15):e523-e661.

16. Dua A, Dang P, Shaker R, Varadarajan P, Pai RG. Barriers to surgery in severe aortic stenosis patients with class I indications for aortic valve replacement. J Heart Valve Dis. 2011;20(4):396-400.

17. Malaisrie SC, Tuday E, Lapin B, et al. Transcatheter aortic valve implantation decreases the rate of unoperated aortic stenosis. Eur $J$ Cardiothorac Surg. 2011;40(1):43-48.
18. Pradelli L, Zaniolo O. Perceval sutureless valves in isolated and concomitant AVR procedures: an economic model shows overall decrease of costs for isolated or combined operations. Farmeconomia. 2012;13(4):159-174.

19. Cohen JW, Monheit AC, Beauregard KM, et al. The Medical Expenditure Panel Survey: a national health information resource. Inquiry. 1996;33(4):373-389.

20. Cohen SB, DiGaetano R, Goksel H. Estimation Procedures in the 1996 Medical Expenditure Panel Survey Household Component. Rockville (MD): Agency for Health Care Policy and Research; 1999. MEPS Methodology Report No. 5. AHCPR Pub. No. 99-0027.

21. Lee DW, Meyer JW, Clouse J. Implications of controlling for comorbid conditions in cost-of-illness estimates: a case study of osteoarthritis from a managed care system perspective. Value Health. 2001;4(4): 329-334.

22. Cragg JG. Some statistical models for limited dependent variables with application to the demand for durable goods. Econometrica. 1971;39(5):829-844

23. Mullahy J. Much ado about two: reconsidering retransformation and the two-part model in health econometrics. J Health Econ. 1998;17(3):247-281.

24. Bureau of Labor Statistics. Consumer Price Index. Available from: http://www.bls.gov/cpi/home.htm. Accessed September 5, 2016.

25. Kennedy P. A Guide to Econometrics. 5th ed. Cambridge (MA): MIT Press; 2003.

26. US Census Bureau, Statistical Abstract of the United States: 2012. Available from: http://www2.census.gov/library/publications/2011/ compendia/statab/131ed/tables/pop.pdf. Accessed September 13, 2016.

27. Enriquez-Sarano M, Akins CW, Vahanian A. Mitral regurgitation. Lancet. 2009;373(9672):1382-1394.

28. van Geldorp MW, van Gameren M, Kappetein AP, et al. Therapeutic decisions for patients with symptomatic severe aortic stenosis: room for improvement? Eur J Cardiothorac Surg. 2009;35(6): 953-957.

29. Gada H, Scuffham PA, Griffin B, Marwick TH. Quality-of-life implications of immediate surgery and watchful waiting in asymptomatic aortic stenosis: a decision-analytic model. Circ Cardiovasc Qual Outcomes. 2011;4(5):541-548.

30. Marumoto A, Nakamura Y, Kishimoto Y, Saiki M, Nishimura M. Optimal timing of aortic valve replacement in elderly patients with severe aortic stenosis. Surg Today. 2014;44(1):84-93.

31. Wu Y, Jin R, Gao G, Grunkemeier GL, Starr A. Cost-effectiveness of aortic valve replacement in the elderly: an introductory study. JThorac Cardiovasc Surg. 2007;133(3):608-613.

32. Brennan JM, Edwards FH, Zhao Y, O'Brien SM, Douglas PS, Peterson ED. Long-term survival after aortic valve replacement among highrisk elderly patients in the United States: insights from the Society of Thoracic Surgeons Adult Cardiac Surgery Database, 1991 to 2007. Circulation. 2012;126(13):1621-1629.

33. Machlin S, Cohen J, Elixhauser A, Beauregard K, Steiner C. Sensitivity of household reported medical conditions in the medical expenditure panel survey. Med Care. 2009;47(6):618-625.

34. Buntin MB, Zaslavsky AM. Too much ado about two-part models and transformation? Comparing methods of modeling Medicare expenditures. J Health Econ. 2004;23(3):525-542. 


\section{Supplementary materials}

Table SI ICD-9 codes for identifying aortic valve disease patients

Aortic valve disease patients identified by any of these primary diagnosis codes

395 Diseases of aortic valve

$395.0 \quad$ Rheumatic aortic stenosis

395.2 Rheumatic aortic stenosis with insufficiency

424.1 Aortic valve disorders

746.3 Congenital stenosis of aortic valve; congenital aortic stenosis

Mitral valve disease patients identified by any of these primary diagnosis codes

$394.0 \quad$ Mitral stenosis

394.9 Other, an unspecified mitral valve disease

424.0 Mitral valve disorders

746.5 Congenital stenosis of mitral valve

746.6 Congenital mitral insufficiency

Abbreviation: ICD, International Classification of Diseases.

Table S2 Methods for stratifying subjects by symptom status

Symptomatic patients defined by the presence of any of these secondary ICD-9 codes

413.xx Angina

$780.2 x \quad$ Syncope

$780.4 \quad$ Dizziness and giddiness

785.I Palpitations

786.0 Dyspnea and respiratory abnormalities

786.02 Orthopnea

786.03 Apnea

$786.05 \quad$ Shortness of breath

$786.07 \quad$ Wheezing

$786.5 x$

Chest pain

Notes: Results also stratified by asymptomatic and symptomatic disease status; asymptomatic patients defined by not having an ICD-9 code listed above.

Abbreviation: ICD, International Classification of Diseases.

ClinicoEconomics and Outcomes Research

\section{Publish your work in this journal}

ClinicoEconomics and Outcomes Research is an international, peerreviewed open-access journal focusing on health technology assessment, pharmacoeconomics and outcomes research in the areas of diagnosis, medical devices, and clinical, surgical and pharmacological intervention. The economic impact of health policy and health systems

\section{Dovepress}

organization also constitute important areas of coverage. The manuscript management system is completely online and includes a very quick and fair peer-review system, which is all easy to use. Visit http://www.dovepress.com/testimonials.php to read real quotes from published authors. 